EXTENSIONS DIEDRALES DE DEGRE $2 \ell$ ( $\ell$ PREMIER $\geqq 5)$

CONTENANT UN CORPS QUADRATIQUE IMAGINAIRE EUCLIDIEN ET ORDRES MONOGENES 
Extensions diédrales de degré $2 \ell$ ( $\ell$ premier $\geq 5)$

contenant un corps quadratique imaginaire euclidien

et ordres monogènes

par Jean COUGNARD

$\S 1 \quad$ Introduction.

Soit $\mathrm{N} / \mathrm{K}$ une extension algébrique de degré fini de corps de nombres ; $\mathbb{Z}_{N}$ et $\mathbb{Z}_{K}$ désignant la clôture intégrale de $\mathbb{Z}$ dans chacun de ces corps, on dit que $\mathbb{Z}_{N}$ est $\mathbb{Z}_{K}$-monogène slil existe un élément $\theta$ de $N$ tel que $\mathbb{Z}_{N}=\mathbb{Z}_{K}[\theta]$. On se propose de démontrer :

Théorème. Soit $K$ un corps quadratique imaginaire dont l'anneau des en tiers $\mathbb{Z}_{K}$ est euclidien et $N / \mathbb{Q}$ une extension diédrale de degré $2 \ell(l$ premier $\geq 5$ ) contenant $K$, alors $\mathbb{Z}_{N}$ n'est pas $\mathbb{Z}_{K}$-monogène, sauf eventuellement pour une des extensions diédrales de degré 14 contenant $Q(j)$ et de conducteur relatif $7^{2} \times 43$.

\section{\$2 Rappels.}

Désignons par $w$ le nombre des racines de llunité contenues dans $K$. Dans un travail précédent [Co] on avait utilisé la méthode développée par M.N. Gras ( [G1], [G2]) pour les extensions abéliennes de $\mathbb{Q}$ et on avait démontré les résultats suivants ( avec les mêmes nota tions ) :

Pour que $\mathbb{Z}_{N}$ soit $\mathbb{Z}_{K}$-monogène il faut :

- soit que $N / K$ soit non ramifiée ,

- soit que $p=w \ell+1$ soit premier et que au plus les idéaux au dessus de $p$ soient ramifiés dans $N / K$,

- soit que $w=4, l=5$ et seuls les idéaux au-dessus de 5 soient ramifiés dans $\mathrm{N} / \mathrm{K}$,

- soit que $w=6, l=7$ et seuls les idéaux au-dessus de 7 ou 43 soient ramifiés dans $\mathrm{N} / \mathrm{K}$.

On va décomposer la démonstration du théorème en deux parties. Dans la première on suppose que le discriminant $\Delta(N / K)$ de $N / K$ est la puissance d'un idéal premier de $\mathbb{Z}$; dans la seconde on suppose que $w=6, l=7$ et $\Delta(N / K)=\left(7^{2} \times 43\right)^{6}$. 
Dans la première partie de la démonstration on a soit $p=w \ell+1$ premier, soit $w \neq 2$ et $l=w+1$ premier. Notons par q le nombre premier égal soit à $p$, soit à $l$ tel que $\Delta(N / K)$ soit une puissance de $q$. On se place dans une situation un peu plus générale que celle de l'énon cé et on fait les hypothèses :

$H 1$ - le nombre de classes de $K$ est premier à $\ell$

H2- les idéaux premiers de $\mathbb{Z}_{K}$ au-dessus de q sont principaux

H3- $K=Q(\sqrt{-d})$, $d$ entier $>0$ avec $d \neq 19$ (24).

Remarquons que les corps quadratiques imaginaires dont l'anneau des entiers est principal sont les $\mathbb{Q}(\sqrt{-d})$ avec $d=1,2,3,7,11,19,43$, 67,163 et que parmi eux seuls ceux où $d \neq 19(24)$ ont un anneau d'en tiers euclidien.

Le discriminant $\Delta(N / K)$ est la puissance $(\ell-1)$-ème d'un idéal f engendré par un élément de $\mathbb{Z}$ et norme dans $\mathrm{N} / \mathrm{K}$ d'un idéal entier ₹ de $N$. Par ailleurs si $\mathbb{Z}_{N}=\mathbb{Z}_{K}[\theta]$, il est facile de démontrer que $\mathcal{F}=(\theta-\sigma(\theta))$ où $\sigma$ désigne un générateur de $\mathrm{Gal}(\mathrm{N} / \mathrm{K})$. Pour démontrer le théorème, il suffit de prouver que ₹'est pas principal et pour cela de constater que le Frobenius de lidéal dans une extension non ramifiée de $\mathrm{N}$ n'est pas réduit à l'élément neutre.

Dans les paragraphes $3,4,5$ on traite le premier cas, liidéal f est engendré par le nombre premier q et les hypothèses $\mathrm{H}_{1}, \mathrm{H} 2, \mathrm{H} 3$ sont supposées vérifiées.

\section{\$3 Problèmes de ramification.}

Lorsque $\mathrm{q}=l=\mathrm{w}+1$ avec $\mathrm{w} \neq 2$, il est évident que $\mathrm{q}$ est décomposé dans $\mathbb{Z}_{K}$. Ecrivons $q \mathbb{Z}_{K}=q_{1} q_{2}$; ces idéaux sont sauvagement ramifiés dans $N / K$ et $\Delta(N / K)=\left(q_{1}^{2} q_{2}^{2}\right)^{l-1}$, le conducteur de $N / K$ est égal à $q_{1}^{2} q_{2}^{2}$ et $N$ est inclus dans le composé des extensions $K^{\left(q_{i}^{2}\right)} / K$ de conducteur $q_{i}^{2}$ et de degré $l$.

Si $p=w l+1$ est premier et $\Delta(N / K)=p^{l-1}$, l'idéal $p$ n'est pas ramifié dans $K / \mathbb{Q}$, sinon il serait totalement et modérément ramifié dans une extension non cyclique. Montrons maintenant que $p$ n'est pas inerte dans $K / \mathbb{Q}$. Si $p$ était inerte dans $K / \mathbb{Q}$, le degré de la $l$-extension de $K$ de conducteur $p$ serait égal à la $l$-partie de $\frac{\varphi(p)}{w}$ ( $\varphi$ indicateur d'Euler généralisé) or $\frac{\varphi(p)}{w}=\ell x(p+1)$. Cette extension est 
composée de $K$ et du sous-corps réel maximal de $Q^{(p)}$, elle est abé lienne sur $\mathbb{Q}$. On peut donc écrire $q \mathbb{Z}_{K}=q_{1} q_{2}$ et $N$ est inclus dans le composé des extensions $k^{\left(q_{i}\right)} / K$ de conducteur $q_{i}$ et de degré $\ell$.

Si $q=l($ resp. $q=p=w l+1)$ on note $K_{i}(i=1,2)$ l'extension de $K$ de degré $l$ et de conducteur $q_{i}^{2}\left(\right.$ resp. $q_{i}$ ) et $L=K_{1} K_{2}$. On constate que $L$ est aussi le composé de $N$ et de $L_{1}$, l'extension cyclique de $\mathbb{Q}$ de degré premier $\ell$ dont le discriminant est une puissance de $q$.

\$4 Démonstration de la non-monogénéité dans le premier cas .

Admettons le lemme suivant dont la démonstration est reportée au $\S 5$.

Lemme 1. Si les hypothèses $H 1, H 2, H 3$ sont vérifiées et si f est une puissance dlun nombre premier de $\mathbb{Z}$, liidéal $q_{1}\left(\right.$ resp. $\left.q_{2}\right)$ est inerte dans $K_{2}\left(\right.$ resp. $\left.K_{1}\right)$.

On peut alors démontrer la proposition :

\section{Proposition 1. Le $\ell$-nombre de classes de $N$ est égal à $l$.}

Démonstration: Si ce nombre était multiple de $l^{2}, l$ diviserait le nombre de classes de $L$. Dlaprès le lemme 1 , un seul idéal est ramifié dans $L / K_{1}$; l'application de la formule des classes ambiges ( [C]) montre que $l$ divise le nombre de classes de $K_{1}$. Comme un seul idéal est ramifié dans $K_{1} / K$ on peut à nouveau appliquer la formule des classes ambiges ce qui donne une contradiction avec l'hypothèse $\mathrm{H} 1$.

Soient $q_{i}^{\prime}$ les idéaux premiers de $\mathbb{Z}_{N}$ au-dessus des idéaux $q_{i}$.

Proposition 2. Les idéaux $q$ ! ont même image dans la $l$-composante du groupe des classes de $\mathbb{Z}_{\mathrm{N}}$.

Démonstration: Soient $H$ le corps de classes de Hilbert de $N$, $\not$ le groupe des classes de $N$. L'application d'Artin induit un isomorphisme entre la $l$-composante de $\#$ et $G a l(L / N)$ qui est celle de $G a l(H / N)$. 
A l'image diun idéal premier $\mathfrak{P}$ de $\mathbb{Z}_{N}$ correspond l'automorphisme de Frobenius $\left(\frac{L / N}{P}\right)$. Les idéaux $q !$ étant inertes dans $L / N$, leurs ima ges dans \& engendrent sa $\ell$-composante. Montrons qu'elles sont égales: Si $\tau$ est un élément d'ordre 2 de $G a l(N / Q)$ on a $\tau q_{1}^{\prime}=q_{2}^{\prime}$ et $\tau$ opère trivialement sur $\mathrm{Gal}\left(\mathrm{L} / \mathrm{N}\right.$ ) par automorphisme intérieur puisque $L=N L_{1}$; on en déduit :

$$
\left(\frac{L / N}{q_{2}^{!}}\right)=\left(\frac{L / N}{\tau q_{1}^{!}}\right)=\tau\left(\frac{L / N}{q_{1}^{!}}\right) \tau=\left(\frac{L / N}{q_{1}^{!}}\right) \text {. }
$$

Par l'hypothèse $\mathrm{H} 2, q$ ! est d'ordre $\ell$ dans $\not$, Jont la classe est le carré ou la puissance quatrième de celle de $q_{i}^{\prime}$ n'est pas principal.

\section{§5 Démonstration du lemme 1.}

L'idéal $q_{1}$ étant principal est décomposé dans le corps de classes de Hilbert de $K$, le degré $l$ de $K_{2} / K$ étant premier au nombre de classes de $K$, pour que $q_{1}$ ne soit pas inerte dans $K_{2} / K$, il faut que $q_{1}$ appartienne au rayon modulo $q_{2}\left(q_{2}^{2}\right.$ si $\left.q=l\right)$.

Si $K=\mathbb{Q}(\sqrt{-d})$ avec - $d \neq 1(4)$, pour que $q_{1}$ appartienne au rayon modulo $q_{2}$ il faut et il suffit que :

$$
q_{1}=(a+b \sqrt{-d}) \quad \text { avec } a+b \sqrt{-d} \equiv 1 \quad(a-b \sqrt{-d}) a, b \in \mathbb{Z}
$$
ce qui implique $(a-1)^{2}+d b^{2} \equiv 0\left(a^{2}+d b^{2}\right)$ avec $q=a^{2}+d b^{2}$ ceci équivaut à $\quad 2 a-1 \equiv 0\left(a^{2}+d b^{2}\right)$.

$a=0$ étant impossible envisageons d'abord $a>0$ la congruence implique $0<\mathrm{a}^{2}<2 \mathrm{a}-1$ ce qui est impossible.

Si $a<0$, en multipliant par -1 et en changeant de notation, on est conduit à $2 a+1 \equiv 0 \quad\left(a^{2}+d b^{2}\right)$ avec $a>0$

ce qui donne $\quad a^{2}<a^{2}+d b^{2} \leq 2 a+1$

ce qui impose $\quad(a-1)^{2}<2$ avec a entier, a $>0$.

On en déduit soit que $a=1$ ce qui donne $q=3$ soit $a=2$ qui donne $q=5$. La condition $p=2 \ell+1$ avec $l$ premier, $l \geq 5$, nous conduit à $q=l=5$, $a=2, b= \pm 1, d=1$. On est alors dans le cas où le conducteur est $q_{2}^{2}$ ce qui conduit aux congruences

$$
2+i \equiv i^{r} \quad\left((2-i)^{2}\right) \quad 0 \leq r \leq 3
$$

dont on vérifie immédiatement qu'aucune n'est satisfaite.

11 résulte de ceci que si $q_{1}$ est décomposé dans $K_{2}$ clest que $-d \equiv 1$ (4) . La condition $q_{1}$ appartient au rayon modulo $q_{2}$ donne : 


$$
a+b \frac{-1+\sqrt{-d}}{2} \equiv 1 \quad\left(a-b \frac{1+\sqrt{-d}}{2}\right) \quad a, b \in \mathbb{Z}
$$

qui conduit à :

$\left(a-1-\frac{b}{2}\right)^{2}+\frac{b d^{2}}{4} \equiv 0 \quad\left(\left(a-\frac{b}{2}\right)^{2}+\frac{d b^{2}}{4}\right)$ avec $q=\left(a-\frac{b}{2}\right)^{2}+\frac{d b^{2}}{4}$

soit $\quad 1-2 a+b \equiv 0 \quad\left(a^{2}-a b+b^{2} \frac{(1+d)}{4}\right)$.

Etudions cette congruence suivant le signe de $1-2 a+b$.

1) Si $1-2 a+b<0$ la congruence s'écrit :

$$
1-2\left(a-\frac{b}{2}\right) \leq-\left(a-\frac{b}{2}\right)^{2}-\frac{d b^{2}}{4}<0
$$

ce qui donne $0<\left(a-\frac{b}{2}\right)^{2}<2\left(a-\frac{b}{2}\right)-1$

soit :

$$
\left[\left(a-\frac{b}{2}\right)-1\right]^{2}<0 \text { ce qui est impossible. }
$$

2) Si $1-2 a+b>0$ on obtient :

(*) $0 \leq\left(a-\frac{b}{2}\right)^{2}<\left(a-\frac{b}{2}\right)^{2}+\frac{d b^{2}}{4} \leq 1-2\left(a-\frac{b}{2}\right)$

d'où $\left(a-\frac{b}{2}\right)^{2}+2\left(a-\frac{b}{2}\right)-1<0$

soit $\quad \frac{-1-\sqrt{5}}{2}<a-\frac{b}{2}<\frac{-1+\sqrt{5}}{2}$

ce qui, joint à la condition de départ $a-\frac{b}{2}<\frac{1}{2}$, donne pour $a-\frac{b}{2}$ les valeurs $-\frac{3}{2},-1,-\frac{1}{2}, 0$. Etudions chacune de ces possibilités:

a) Si $a-\frac{b}{2}=0$ les inégalités $(*)$ donnent $0<\mathrm{db}^{2} \leq 4$ qui joint à $d \equiv 3(4)$ impose $d=3, b^{2}=1$ ce qui est impossible puisque $a-\frac{b}{2}=0$ implique la parité de $b$.

b) Si $a-\frac{b}{2}=-\frac{1}{2}$, b est impair et les inégalités (*) donnent $0<d b^{2}<7$ ce qui joint à $d \equiv 3$ (4) conduit à $b^{2}=1, d=3$ ou 7 . Pour chacun de ces cas on a

- a) $b=1, d=3$ alors $a=0$ et $a+b w=\frac{-1+\sqrt{-3}}{2}$ qui est une unité et donc n'engendre pas un idéal premier.

- B) $b=-1, d=3$ alors $a=-1, a+b w=-1-\frac{-1+\sqrt{-3}}{2}$ est encore une unité . 
- y) $b=1, d=7$ alors $a=0, a+b w=\frac{-1+\sqrt{-7}}{2}$ dont la norme 2 devrait être un nombre premier impair.

- 8) $b=-1, d=7$ alors $a=-1, a+b w=-1-\frac{-1+\sqrt{-7}}{2}$ a encore pour norme 2 .

c) Si $a-\frac{b}{2}=-1$ les inégalités $\left(^{\star}\right)$ deviennent $0<d b^{2} \leq 8$ on trouve $d=3$ ou 7 ce qui ne convient pas du fait de la parité de b.

d) Si $a-\frac{b}{2}=-\frac{3}{2}$, b est impair, les inégalités $(\star)$ deviennent $0<d b^{2} \leq 7$ ce qui impose $b^{2}=1, d=3$ ou 7 .

- a) $b=1, d=3$ alors $a=-1, a+b w=-1+\frac{-1+\sqrt{-3}}{2}$

qui donne $q=3$ ce qui est exclus.

- B) $b=-1, d=3$ alors $a=-2, a+b w=-2-\frac{-1+\sqrt{-3}}{2}$ qui donne aussi $q=3$.

- $\gamma) b=1, d=7$ alors $a=-1, a+b w=-1+\frac{-1+\sqrt{-7}}{2}$ dont la norme est 4 alors que ce devrait être un nombre premier impair. ła norme est également 4 .

- 8) $b=-1, d=7$ alors $a=-2, a+b w=-2-\frac{-1+\sqrt{-7}}{2}$ dont 3) Si $1-2 a+b=0$, b est impair et $a=\frac{b+1}{2}$, on obtient $a+b \omega=\frac{1+b \sqrt{-d}}{2}$ avec $b$ impair. Le nombre premier $q$ est égal à $\frac{1+d b^{2}}{4}$

a) Si $d=3, q=7=\ell, b= \pm 3$ ce qui donne les idéaux de $Q(j)$ au-dessus de 7 . Dans ce cas le conducteur est $q_{2}^{2}$, il faut, en fait, regarder les congruences $\frac{1+3 \sqrt{-3}}{2} \equiv(-j)^{r}\left(\left(\frac{1-3 \sqrt{-3}}{2}\right)^{2}\right)$, $0 \leq r \leq 5$, dont on vérifie qu'elles n'ont pas de solution.

b) Il reste à envisager $q=p=2 \ell+1$ ce qui nous donne : $d b^{2}=8 l+3$, comme $b$ est impair, on en déduit $d \equiv 3(8)$. Si on regarde l'égalité modulo 3 :

$\mathrm{db}^{2} \equiv 2 \ell$ (3), comme $l \geq 5$ on $\mathrm{a} b \neq 0$ (3). Par conséquent : $\mathrm{d} \equiv 2 \ell$ (3); comme $2 \ell+1$ est premier $l \equiv 2$ (3).

On obtient facilement $d \equiv 3$ (8) et $d \equiv 1$ (3) soit $d \equiv 19$ (24) ce qui est interdit par l'hypothèse $\mathrm{H}_{3}$.

Pour terminer la démonstration du théorème, il reste à étudier les extensions diédrales de degré 14 contenant $Q(j)=K$, le conducteur de $\mathrm{N} / \mathrm{K}$ étant $7^{2} \times 43$. 
$\S 6$ Extensions diédrales imaginaires de degré 14 et de discriminant $3^{7}\left(7^{2} \times 43\right)^{12}$

$$
\text { On écrit } \begin{aligned}
7 \mathbb{Z}[j]=\mathcal{L}_{1} \mathcal{L}_{2} \text { avec } \mathcal{L}_{1}=(1-2 j), \mathcal{L}_{2}=(3+2 j) \\
43 \mathbb{Z}[j]=p_{1} p_{2} \text { avec } p_{1}=(1-6 j), p_{2}=(7+6 j)
\end{aligned}
$$

et on considère les corps $k^{\left(\Sigma_{1}^{2}\right)}, K^{\left(\Sigma_{2}^{2}\right)}, k^{\left(p_{1}\right)}, K^{\left(p_{2}\right)}$; le composé $L$ de ces corps est une extension abélienne de degré $7^{4}$ de $Q(j)$ dont le groupe de Galois est du type $(7,7,7,7)$, clest le corps des genres des extensions diédrales de $Q$, et de discriminant $3^{7}\left(7^{2} \times 43\right)^{12}$. Soit $N$ une telle extension, on note $p_{1}^{\prime}$ (resp. $p_{2}^{\prime}, \delta_{1}^{\prime}, \Sigma_{2}^{\prime}$ ) liidéal de $\mathbb{Z}_{N}$ au dessus de $p_{1}$ (resp. $\left.p_{2}, \mathcal{L}_{1}, \mathcal{\Sigma}_{2}\right)$, pour démontrer que $\mathbb{Z}_{N}$ 'est pas $Z_{K}$-monogène il suffit de démontrer que l'idéal ambige $1=\left(\delta_{1} \Sigma_{2}\right)^{2} p_{1}^{\prime} p_{2}^{\prime}$ n'est pas principal. Le principe de la démonstration reste le même ; on considère llimage de I par llapplication d'Artin dans le groupe de Galois de l'extension $H / N$ (où $H$ est le corps de classes de Hilbert de $N$ ). L'idéal l est principal si et seulement si cette image est l'élément neutre et on a un premier renseignement en étudiant sa restriction à $L$.

\section{Lemme - L'idéal I n'est principal que pour au plus un corps}

Démonstration: La restriction à $L$ de l'image d'un idéal premier $\mathfrak{P}$ par l'application d'Artin dans $\mathrm{Gal}(\mathrm{H} / \mathrm{N})$ est égale à l'automorphisme de Frobenius $\left(\frac{L / N}{P}\right)$. II suffit donc de démontrer que $\left(\frac{L / N}{\sum_{1}^{2} \sum_{2}^{2} p_{1}^{\prime} p_{2}^{!}}\right) \neq 1$.

Précisons tout d'abord la structure de $G a l(L / K)$. Soit $I_{p_{1}}$ (resp. $\left.I_{p_{2}}, I_{\delta_{1}}, I_{\Sigma_{2}}\right)$ le groupe dinertie de $p_{1}\left(\right.$ resp. $\left.p_{2}, \Sigma_{1}, \delta_{2}\right)$ dans $\operatorname{Gal}(L / K)$ et $\sigma_{1}\left(\right.$ resp. $\left.\sigma_{2}, \nu_{1}, \nu_{2}\right)$ un générateur de $I_{p_{1}}$ (resp. $\left.{ }^{\prime} p_{2}, I_{\Sigma_{1}}, I_{\delta_{2}}\right)$. On peut fixer sans ambiguité ces générateurs. On sait que $\delta_{2}$ est inerte dans $K^{\left(\Sigma_{1}^{2}\right)}$. On pose donc :

$$
\nu_{1 / K}\left(\Sigma_{1}^{2}\right)=\left(\frac{K^{\left(\Sigma_{1}^{2}\right)} / K}{\Sigma_{2}}\right) \text { et } \nu_{2}\left(\Sigma_{2}^{2}\right)=\left(\frac{K^{\left(\Sigma_{2}^{2}\right)} / K}{\delta_{1}}\right) \text {. }
$$


Rappelons que la conjugaison complexe $\tau$ opère sur Gal (L/N) et sur le groupe des idéaux fractionnaires de $K$; les propriétés fonctorielles de l'application d'Artin montrent que $\tau \nu_{1} \tau=\nu_{2}$.

$$
\begin{aligned}
& \text { De même } p_{2} \text { est inerte dans } K^{\left(p_{1}\right)} / K \text {, on pose : } \\
& \sigma_{1 / K}\left(p_{1}\right)=\left(\frac{K^{\left(p_{1}\right)} / K}{p_{2}}\right) \text { et } \sigma_{2}\left(p_{2}\right)=\left(\frac{K^{\left(p_{2}\right)} / K}{p_{1}}\right)
\end{aligned}
$$

on en déduit $\tau \sigma_{1} \tau=\sigma_{2}$.

On peut vérifier sans trop de difficulté les congruences suivantes:

$$
\begin{aligned}
& (3+2 j)^{5} \equiv-(1-6 j)\left(\Sigma_{1}^{2}\right) \quad(3+2 j)^{3} \equiv-j^{2}(7+6 j)\left(\Sigma_{1}^{2}\right) \\
& (7+6 j)^{3} \equiv-j^{2}(1-2 j)\left(p_{1}\right) \quad(7+6 j)^{4} \equiv-(3+2 j) \quad\left(p_{1}\right)
\end{aligned}
$$

D'où l'on déduit :

$$
\begin{aligned}
& \left(\frac{K^{\left(\Sigma_{1}^{2}\right)} / K}{p_{1}}\right)=\nu_{1 / K}^{5}\left(\Sigma_{1}^{2}\right) ;\left(\frac{K^{\left(\Sigma_{1}^{2}\right)} / K}{p_{2}}\right)=\nu_{1 / K}^{3}\left(\Sigma_{1}^{2}\right) ; \\
& \left(\frac{K^{\left(p_{1}\right)} / K}{\Sigma_{1}}\right)=\sigma_{1 / K}^{3}\left(p_{1}\right) ;\left(\frac{K^{\left(p_{1}\right)} / K}{\delta_{2}}\right)=\sigma_{1 / K}^{4}\left(p_{1}\right)
\end{aligned}
$$

et au moyen de la conjugaison par $T$ :

$$
\begin{aligned}
& \left(\frac{K^{\left(\Sigma_{2}^{2}\right)} / K}{p_{2}}\right)=\nu_{2 / K}^{5}\left(\Sigma_{2}^{2}\right) ;\left(\frac{K^{\left(\Sigma_{2}^{2}\right)} / K}{p_{1}}\right)=\nu_{2 / K}^{3}\left(\Sigma_{2}^{2}\right) ; \\
& \left(\frac{K^{\left(p_{2}\right)} / K}{\Sigma_{2}}\right)=\sigma_{2 / K}^{3}\left(p_{2}\right) ;\left(\frac{K^{\left(p_{2}\right)} / K}{\Sigma_{1}}\right)=\sigma_{2 / K}^{4}\left(p_{2}\right)
\end{aligned}
$$

On est maintenant à même de déterminer les groupes de décomposition des idéaux $p_{i}$ et $\mathcal{L}_{i}$ dans $L / K$. On pourra alors déterminer les automorphismes de Frobenius des idéaux $p_{i}, \Sigma_{i}$ au-dessus de $p_{i}, \mathcal{L}_{i}$ dans $\operatorname{Gal}(L / N)$. Soit $D_{\mathcal{L}_{1}}$ le groupe de décomposition de $\mathcal{L}_{1}$ dans Gal $\left(L / K^{\left(\Sigma_{1}^{2}\right)}\right)$; pour tout corps $M, K \subset M \subset L$, tel que $\mathcal{L}_{1}$ ne soit pas ramifié dans $M / K$ et $\mu=\left(\frac{N / K^{\left(\Sigma_{1}^{2}\right)}}{I_{1}}\right)$ où $I_{1}$ est liidéal premier de $K^{\left(\Sigma_{1}^{2}\right)}$ 
au-dessus de $\mathcal{L}_{1}$, on a :

$$
\mu / M=\left(\frac{M / K}{N_{K}\left(\Sigma_{1}^{2}\right)_{/ K}\left(I_{1}\right)}\right)=\left(\frac{M / K}{\Sigma_{1}}\right)
$$

On en déduit donc $\mu_{K}\left(\Sigma_{2}^{2}\right)=v_{2}, \mu /{ }_{K}\left(p_{1}\right)=\sigma_{1}^{3} ; \mu /\left(p_{2}\right)=\sigma_{2}^{4}$ et par conséquent $\mu=\nu_{2} \sigma_{1}^{3} \sigma_{2}^{4}$. Le groupe $D_{\mathcal{L}_{1}}$ est donc engendré par $\nu_{1}$ et $\nu_{2} \sigma_{1}^{3} \sigma_{2}^{4}$. Par conjugaison le groupe de décomposition $D_{\Sigma_{2}}$ de $\Sigma_{2}$ dans $L / K$ est engendré par $\nu_{2}$ et $\nu_{1} \sigma_{1}^{4} \sigma_{2}^{3}$.

Procédons de la même manière avec $p_{1}$. Soient $\mathfrak{P}_{1}$ l'idéal au dessus de $p_{1}$ dans $k^{\left(p_{1}\right)}$ et $\mu=\left(\frac{L / K^{\left(p_{1}\right)}}{\mathfrak{P}_{1}}\right)$, pour tout corps $M$, $K \subset M \subset L$, tel que $p_{1}$ ne soit pas ramifié dans $M / K$, on a :

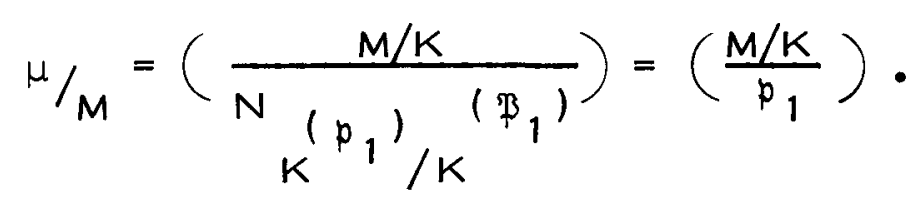

On a donc:

$\mu /_{K}\left(p_{2}\right)=\sigma_{2}, \mu /_{K}\left(\Sigma_{1}^{2}\right)=\nu_{1}^{5}, \mu /_{K}\left(\Sigma_{2}^{2}\right)=\nu_{2}^{3}$ d'où $\mu=\sigma_{2} \nu_{1}^{5} \nu_{2}^{3}$. Le groupe $D_{p_{1}}$ est engendré par $\sigma_{1}$ et $\sigma_{2} \nu_{1}^{5} \nu_{2}^{3}$; par conjugaison le groupe $D_{p_{2}}$ est engendré par $\sigma_{2}$ et $\sigma_{1} \nu_{1}^{3} \nu_{2}^{5}$.

Le composé des extensions diédrales de $\mathbb{Q}$ contenues dans $L$ est le sous-corps de $L$ invariant par $\sigma_{1} \sigma_{2}$ et $\nu_{1} \nu_{2}$; l'extension diédrale dont le conducteur sur $K$ est $7^{2}$ (resp. 43 ) est également invariante par $\sigma_{1}, \sigma_{2}$ (resp. $\left.\nu_{1}, \nu_{2}\right)$; le groupe de Galois de $L$ sur cette ex tension est engendré par $\nu_{1} \nu_{2}, \sigma_{1} \sigma_{2}, \sigma_{1} \sigma_{2}^{-1}$ (resp. $\sigma_{1} \sigma_{2}, \nu_{1} \nu_{2}$, $\nu_{1} \nu_{2}^{-1}$ ). Il y a donc six extensions diédrales de degré 14 , contenant $Q(j)$ et dont le conducteur sur $Q(j)$ est $7^{2} .43$; ce sont les corps $N_{i}$ où $N_{i}$ est le sous-corps de $L$ invariant par $\sigma_{1} \sigma_{2}, \nu_{1} \nu_{2}, \nu_{1} \nu_{2}^{-1} \sigma_{1}^{i} \sigma_{2}^{-i}$ $(1 \leq i \leq 6)$. 
Soit $P$ un des idéaux $p_{r}, \mathcal{L}_{s}$ et $P^{\prime}$ l'idéal premier de $N_{i}$ audessus de $P$. Pour tout corps $M, K \subset M \subset L$, tel que $P$ ne soit pas ramifié dans $M / K$, on a $\left(\frac{L / N_{i}}{P^{\prime}}\right)_{M}=\left(\frac{M / K}{N_{N_{i} / K}\left(P^{\prime}\right)}\right)=\left(\frac{M / K}{P}\right)$. Si on écrit $\left(\frac{L / N_{i}}{\Sigma !}\right)=\mu=\left(\nu_{1} \nu_{2}\right)^{a}\left(\sigma_{1} \sigma_{2}\right)^{b}\left(\nu_{1} \nu_{2}^{-1} \sigma_{1}^{i} \sigma_{2}^{-i}\right)^{c}$ on $a: \mu_{K}\left(\Sigma_{2}^{2}\right)=\nu_{2}$ donc $a-c=1, \mu_{K}\left(p_{1}\right)=\sigma_{1}^{3}$ donc $b+i c=3$ $\mu /\left(p_{2}\right)=\sigma_{2}^{4}$ donc $b-i c=4$

il en résulte que $b=0, c=3 i^{*}, a=1+3 i^{*}$ où $i^{*}$ est l'inverse de $i$ modulo 7 . Par conséquent :

$$
\left(\frac{L / N_{i}}{\mathcal{L} i}\right)=\nu_{1}^{1-i^{*}} \nu_{2} \sigma_{1}^{3} \sigma_{2}^{4} \text { et }\left(\frac{L / N_{i}}{\Sigma_{2}}\right)=\nu_{1} \nu_{2}^{1-i *} \sigma_{1}^{4} \sigma_{2}^{3} \text {. }
$$
De la même manière, si $\mu=\left(\frac{L / N_{i}}{p_{1}^{\prime}}\right)=\nu_{1}^{a+c} \nu_{2}^{a-c} \sigma_{1}^{b+i c} \sigma_{2}^{b-i c}$ on a $\mu_{K}\left(\Sigma_{1}^{2}\right)=\nu_{1}^{5}$ donc $a+c=5 ; \mu / \underset{K}{\left(\Sigma_{2}^{2}\right)}=\nu_{2}^{3}$ donc $a-c=3$ $\mu_{K}\left(p_{2}\right)=\sigma_{2}$ donc $b-i c=1$,

il en résulte $a=4, c=1, b=1+i$ et :

$$
\left(\frac{L / N_{i}}{p_{1}^{!}}\right)=\nu_{1}^{5} \nu_{2}^{3} \sigma_{1}^{1+2 i} \sigma_{2} \text { et }\left(\frac{L / N_{i}}{p_{2}^{!}}\right)=\nu_{1}^{3} \nu_{2}^{5} \sigma_{1} \sigma_{2}^{1+2 i} \text {. }
$$

L'automorphisme de Frobenius associé à $\left(\delta_{1}^{1} \delta_{2}^{1}\right)^{2} p_{1}^{1} p_{2}^{\prime}$ est :

$$
\nu_{1}^{5-2 i^{*}} \nu_{2}^{5-2} i^{*} \sigma_{1}^{2+2 i} \sigma_{2}^{2+2 i}
$$

il est nul si et seulement si $i=6$.

Ce qui donne au plus un corps pour lequel l'idéal I peut être principal. Remarquons que : 


$$
\left(\frac{L / N_{6}}{p_{1}^{\prime}}\right)=\left(\frac{L / N_{6}}{\sum_{2}^{\prime}}\right)^{5} \text { et }\left(\frac{L / N_{6}}{\Sigma_{1}^{\prime}}\right)=\left(\frac{L / N_{6}}{p_{2}^{!}}\right)^{3}
$$

et qu'il y a donc des classes ambiges dans le genre principal.

[C] C. CHEVALLEY : Sur la théorie du corps de classes dans les corps finis et les corps locaux. J. of the Fac. of Sc., Tokyo, Vol.ll, Part 9 (1933).

[Co] J. COUGNARD : Sur la monogénéité de l'anneau des entiers d'une extension diédrale imaginaire de degré $2 p(p$ premier $\geq 5)$.

Soumis à publication.

[G1] M.-N. GRAS : Non monogénéité de l'anneau des entiers des extensions cycliques de $\mathbb{Q}$ de degré premier $\ell \geq 5$. A paraître dans J. of Number Theory.

[G2] M.-N. GRAS : Non monogénéité de l'anneau des entiers de certaines extensions abéliennes de $\mathbb{Q}$. Publ. Math. Fac. Sc. Besançon 1983-84.

\title{
Addenda
}

Dans la démonstration du lemme $1(\$ 5)$ un cas a été oublié au point 3). II faut ajouter :
c) Si $d=3, q=6 l+1$ l'égalité de $q$ avec $\frac{1+d b^{2}}{4}$ avec b impair conduit à $\frac{b-1}{2} \cdot \frac{b+1}{2}=2 \ell$ ce qui est impossible avec $l \geq 5$.

\author{
Jean COUGNARD \\ U. A. 741 CNRS \\ Faculté des Sciences \\ 25030 Besançon Cedex \\ (France)
}

